\title{
Les titres d'articles de presse au confluent de plusieurs formes de discours rapporté
}

\section{Grégoire Lacaze}

\section{Q OpenEdition \\ 1 Journals}

Édition électronique

URL : http://journals.openedition.org/esa/709

DOI : 10.4000/esa.709

ISSN : 2650-2623

\section{Éditeur}

Société de stylistique anglaise

\section{Édition imprimée}

Date de publication : 1 décembre 2016

Pagination : 29-47

ISBN : 978-2-36442-075-5

ISSN : 2116-1747

\section{Référence électronique}

Grégoire Lacaze, "Les titres d'articles de presse au confluent de plusieurs formes de discours rapporté », Études de stylistique anglaise [En ligne], 10 | 2016, mis en ligne le 19 février 2019, consulté le 23 avril 2019. URL : http://journals.openedition.org/esa/709; DOI : 10.4000/esa.709 


\title{
Les titres d'articles de presse au confluent de plusieurs formes de discours rapporté
}

\author{
Grégoire LACAZE \\ Aix Marseille Univ, LERMA, Aix-en-Provence, France
}

\section{Introduction}

L'analyse des discours produits par la presse, menée par les chercheurs en sciences du langage, en sciences de l'information et de la communication et par les spécialistes des médias, s'attache à décrire les pratiques discursives adoptées par les journalistes.

L'article de presse, production textuelle emblématique du discours journalistique, exploite le "contrat de communication médiatique" (Charaudeau 2005, 70) liant un journal à son lectorat. L'«enjeu de captation" (Charaudeau 2006, 32) est tout particulièrement à l'œuvre dans les titres d'articles de presse, qui ont un pouvoir fondamental de séduction du lecteur.

Connaissant une grande variabilité compositionnelle, ces titres sont souvent le lieu privilégié d'émergence de fragments citationnels. L'étude de ces derniers interroge alors la définition même et donc l'identité des formes de discours rapporté.

À partir de considérations syntaxiques, sémantiques et stylistiques, cette recherche exploite un corpus rassemblant des titres d'articles de la presse britannique contemporaine. Elle tente de montrer que certaines constructions, identifiables à du discours direct au niveau formel, ont un statut énonciatif plus ambivalent et indéterminé que leur composition structurelle pourrait laisser croire.

Cette étude s'intéresse ainsi à l'ordre sujet-verbe dans les incises de discours direct sous l'influence de contraintes sémantiques et pragmatiques, notamment par la prise en compte du sémantisme du verbe. 
Elle montre également que la présence d'un locuteur métonymique dans le segment contextualisant illustre la valeur médiative de l'énoncé tout en soulignant l'instabilité syntaxique de l'incise.

Elle tend enfin à mettre en évidence que ces énoncés, qui présentent des schèmes de récurrence dans leur composition, se trouvent en fait localisés au confluent de plusieurs plans énonciatifs suivant la composition intrinsèque du segment contextualisant présent dans le titre d'article. L'instabilité énonciative caractérisant ces titres peut les positionner sur une zone de convergence entre plusieurs formes de discours rapporté.

\section{Les titres d'articles de presse à l'étude}

La présente étude fait suite à une précédente recherche (Lacaze 2016) fondée sur la prise en charge, la responsabilité et l'endossement ${ }^{1}$ énonciatifs des titres d'articles de presse. Elle envisage des énoncés présents dans les titres et sous-titres d'articles dans lesquels il est possible d'associer un contenu propositionnel à une source énonciative. Dans cette étude, seuls sont pris en compte les énoncés dans lesquels la source énonciative mentionnée émerge au sein d'un "segment contextualisant $"^{2}$ (Lacaze 2011,36) dont l'extension se réduit à une incise de discours rapporté en position finale. Cette incise est constituée d'un syntagme nominal sujet, qui désigne la source énonciative, et d'un verbe introducteur qui assure la fonction d'insertion du contenu propositionnel associé à cette source énonciative.

Dans cette étude, la composition de base du titre, lorsqu'elle s'identifie à un énoncé de discours direct (DD) au niveau formel, peut se schématiser ainsi :

contenu propositionnel $P$, (syntagme nominal $X$, verbe introducteur $Y$ )

Le verbe introducteur est le plus souvent à mode fini et le temps grammatical est habituellement le présent simple, mais des énoncés au

L'endossement énonciatif envisage le degré d'intervention du locuteur rapporteur dans les propos qu'il rapporte.

2 Le segment contextualisant désigne le segment textuel qui assure la mise en relation d'un contenu propositionnel avec une origine énonciative. 
prétérit ou au Present Perfect sont aussi possibles dans ce contexte énonciatif.

Un titre, dans sa composition canonique, contient un syntagme nominal (SN) $X$ désignant un locuteur animé humain et le verbe say $Y$, verbe de dire par excellence :

(1) Cut length of school holidays, says Michael Gove ${ }^{3}$ (Telegraph.co.uk $18 / 04 / 2013$ )

Le locuteur rapporté qui assume la prise en charge du contenu propositionnel dans cette occurrence de DD est Michael Gove, ministre de l'éducation du gouvernement Cameron de 2010 à 2014. Cet énoncé, qui emprunte la forme d'une "aphorisation " (Lacaze 2015), contient une incise en position finale dans laquelle le verbe say est antéposé, comme c'est le cas le plus souvent quand l'incise contient un anthroponyme.

La structure de l'énoncé devient donc :

contenu propositionnel $P,(Y=$ says, $X=$ Michael Gove $)$

Lorsque le verbe est à mode non fini, le verbe est à l'infinitif (avec TO) :

(2) Gender-selective abortion is illegal, Health Secretary Jeremy Hunt to announce (Telegraph.co.uk 22/05/2014)

La diathèse active est la construction la plus répandue mais la diathèse passive est possible dans le titre d'un article de presse :

(3) Find cheapest rail fares yourself, passengers told (Telegraph.co.uk 28/08/2015)

Dans ce cas, il y a l'ellipse de la copule $B E$ dans toutes les incises figurant dans les titres.

C'est nous qui soulignons dans les énoncés.

L'expression est empruntée à Maingueneau $(2012,22)$ qui la définit ainsi : "L'énoncé détaché n'est pas un fragment de texte, il relève d'un régime d'énonciation spécifique, que nous appellerons aphorisation". 


\section{L'ordre sujet-verbe dans l'incise à la croisée de contraintes sémantiques et pragmatiques}

La composition du segment contextualisant dans un titre d'article influe sur l'ordre sujet-verbe (Lacaze 2013, 2016). En effet, la conjonction du sémantisme du verbe introducteur et du type de source énonciative peut parfois conditionner l'ordre préférentiel de la séquence $(X, Y)$ au sein du segment contextualisant.

\section{Le verbe say, parangon du verbe introducteur}

Quand le sujet est un animé humain désigné par un anthroponyme, l'inversion est souvent adoptée avec le verbe say mais celle-ci n'est pas obligatoire, comme dans l'énoncé suivant qui conserve sa grammaticalité :

(4) Tories need to speak the language of the working classes, Liam Fox says (Telegraph.co.uk 07/05/2013)

L'homme politique conservateur Liam Fox, ancien ministre de la défense, exprime un point de vue sur la ligne politique que son parti devrait adopter.

Avec le verbe say, l'inversion semble favorisée dans la presse pour des raisons sémantiques : l'identité de la source énonciative est dévoilée en fin d'énoncé, ce que rend possible l'inversion sujet-verbe, le verbe say étant aussi relativement pauvre au niveau sémantique.

Dans l'énoncé ci-dessous, alors que le titre contient l'anthroponyme Ken Livingstone avec l'ordre canonique sujet-verbe dans l'incise, le sous-titre contient une fonction définitoire du sujet animé humain (former London Mayor en référence à son mandat politique) associée à l'inversion sujet-verbe :

(5) Jews vote Tory because they are rich, Ken Livingstone says (Telegraph.co.uk 07/05/2014)

(6) Jewish people have switched from Labour to Conservative as they have got richer over the past half century, says former London Mayor (Telegraph.co.uk 07/05/2014)

La présence d'une fonction définitoire du locuteur cité semble orienter vers une construction syntaxique de l'incise dans laquelle apparaît l'inversion sujet-verbe. Cette fonction définitoire peut également être la mention d'un grade ou d'un titre de noblesse : 
(7) Wind farms are useless, says Duke of Edinburgh (Telegraph.co.uk $19 / 11 / 2011$ )

En l'absence d'anthroponyme, la mention isolée de la fonction du locuteur cité suffit pour déclencher l'inversion :

(8) Electric cars will become as ubiquitous as the internet, says transport minister (Telegraph.co.uk 22/01/2016)

L'absence de déterminant dans les titres et les sous-titres est un élément favorisant la construction syntaxique avec l'inversion.

Toutefois, quand la fonction accompagne l'anthroponyme, l'inversion demeure facultative :

(9) Cougars on the rise because women look better in their 40s, actress Helen McCrory says (Telegraph.co.uk 14/04/2013)

(10) Bahrain protesters are just like those 'complaining about Mrs Thatcher', says F1 chief Bernie Ecclestone (Telegraph.co.uk 15/04/2013)

Ainsi, lorsque la fonction définitoire est mentionnée seule, l'inversion sujet-verbe est de mise. Lorsqu'elle accompagne la mention de l'anthroponyme, le rapporteur peut avoir le choix de l'ordre syntaxique sujet-verbe dans l'incise.

Toutefois, l'inclusion dans le segment contextualisant de précisions référentielles sur le locuteur cité favorise l'inversion :

(11) 'Everything seemed okay', says father whose son died in suspected teenage suicide pact (Telegraph.co.uk 19/06/2013)

La proposition relative déterminative qui explicite le nom father impose l'inversion en raison d'un "poids sémantique " fort et de sa large extension.

L'ajout au segment contextualisant d'une information concernant la localisation temporelle de l'acte de parole dans la chronologie historique justifie aussi l'inversion :

(12) 'I'm looking forward to it,' says Jeremy Corbyn ahead of predicted victory in Labour leadership (Telegraph.co.uk 10/09/2015) 


\section{Autres verbes introducteurs : le cas du verbe warn}

La diversité des verbes introducteurs pouvant potentiellement introduire des discours cités dans les titres d'articles de presse mériterait une étude dédiée. En effet, il serait intéressant d'analyser le degré de couplage existant entre le sémantisme du syntagme nominal et le sémantisme du verbe introducteur. Dans l'approche méthodologique adoptée ici, c'est le sémantisme du syntagme nominal présent dans l'incise introductrice qui guide l'analyse.

Envisageons le cas d'un verbe à valeur illocutoire comme le verbe warn pour lequel des règles d'usage semblent s'imposer au locuteur rapporteur. Austin (1962) envisage ce verbe comme un verbe illocutoire ("illocutionay verb») sans que son effet perlocutoire soit forcément atteint. Dans son ouvrage How To Do Things with Words, le philosophe du langage propose une taxonomie fine des verbes illocutoires. Le verbe warn y apparait dans la classe des "exercitive verbs" (Austin 1962, 155). Cette valeur est définie ainsi : "the exercitive is an assertion of influence or exercising of power" (Austin 1962, 162).

Les deux structures de base que l'on rencontre pour les titres d'articles sont :

contenu propositionnel $P,(Y=$ warn, $X)$

contenu propositionnel $P,(X, Y=$ warn $)$

Les conditions d'apparition de l'une ou l'autre structure dépendent étroitement de la composition du SN $X$.

Avec la mention d'un anthroponyme dans $X$, qui présente l'identité du locuteur rapporté, l'inversion est privilégiée :

(13) Mothers risk frustration by staying at home, warns Nicole Kidman (Telegraph.co.uk 01/02/2016)

Il en va de même quand la fonction du locuteur cité est mentionnée :

(14) Time running out for China on capital flight, warns bank chief (Telegraph.co.uk 01/02/2016)

Le nombre grammatical du SN $X$ influence notablement l'ordre sujet-verbe. En effet, lorsque le $\mathrm{SN} X$ est à nombre pluriel, l'ordre canonique est préservé : 
(15) 'Shocking delay' into HBOS probe needs thorough response, MPs warn (Telegraph.co.uk 03/02/2016)

(16) Cuts to teachers' pay threatening education, unions warn (Telegraph.co.uk 11/01/2016)

(17) UK weather: Big freeze could follow rain deluge, forecasters warn (Telegraph.co.uk 27/01/2016)

La présence d'un "locuteur métonymique " (Lacaze 2016) comme le nom d'un pays (Poland) ou le nom d'une ville (Strasbourg) ou plus généralement l'usage d'une "métonymi[e] du lieu [qui] abond[e] dans les titres pour donner une résonance accrue aux événements" (Bonhomme 1998,55) concourent à un titre dans lequel l'inversion apparaît :

(18) Brexit will trigger collapse of EU, warns Poland (Telegraph.co.uk 18/01/2016)

(19) Newspaper rounds may breach children's rights, warns Strasbourg (Telegraph.co.uk 27/01/2016)

Toutefois, si le nom du pays se présente sous la forme d'un acronyme, l'ordre canonique est souvent utilisé :

(20) North Korea 'preparing for space launch,' US warns (Telegraph.co.uk 28/01/2016)

(21) Europe must strengthen its anti-terror laws to fight Syrian threat, US warns (Telegraph.co.uk 09/07/2014)

Si l'acronyme précède un nom, c'est l'inversion qui est favorisée :

(22) UK defence cuts will have profound effect, warns US diplomat (Telegraph.co.uk 05/04/2015)

Si l'acronyme désigne une association ou une organisation, un degré de liberté apparaît dans l'ordre sujet-verbe, l'ordre sujet-verbe étant à l'appréciation du locuteur rapporteur :

(23) 'Drowning' oil market is here to stay, warns IEA (Telegraph.co.uk 19/01/2016) 
LES TITRES D'ARTICLES DE PRESSE AU CONFLUENT DE PLUSIEURS

FORMES DE DISCOURS RAPPORTÉ

(24) Budget 2016: Chancellor's avalanche of business taxes has pushed UK to a 'tipping point', CBI warns (Telegraph.co.uk 15/02/2016)

Les quelques énoncés étudiés ici montrent la complexité et la subtilité des règles d'emploi des deux structures de base, qui doivent tenir compte du sémantisme du verbe introducteur $Y$ mais aussi de la valeur sémantique du SN $X$.

\section{L'instabilité syntaxique de l'incise sous l'influence d'un locuteur métonymique}

Un certain degré d'instabilité syntaxique de l'incise voit le jour quand le sujet du verbe d'incise désigne un locuteur métonymique non humain, qui n'est pas en mesure de verbaliser un acte de parole. C'est le cas lorsque le contenu propositionnel $P$, qui s'identifie à un discours cité dans la structure canonique $d u$ discours direct mentionnée précédemment, désigne notamment une production textuelle ou statistique, même si des locuteurs humains en sont à l'initiative. Une enquête, une étude, un rapport, un sondage, des données statistiques, etc. fournissent une information dont le contenu propositionnel est communiqué aux lecteurs en empruntant une construction syntaxique qui mime celle d'un discours direct authentique. Dans de tels énoncés avec un locuteur métonymique, le discours cité n'est généralement pas encadré de guillemets: c'est une forme syntaxique identifiable à du «DD mimétique $\|^{5}$, selon De Mattia-Viviès $(2010,165)$, qui propose cette terminologie à partir de l'étude d'un corpus littéraire sans spécifier le type de locuteur. Avec le DD mimétique, "on se rapproche du discours non rapporté, du discours en direct, par l'absence de guillemets " (De Mattia-Viviès 2010, 165).

\section{Le "dévoiement " du verbe say : l'incise à valeur médiative}

Quand le verbe say suit le contenu propositionnel antéposé, ce verbe perd son sémantisme de base de verbe de parole. Le segment contextualisant est alors réduit à sa valeur "médiative". Selon

5 Par souci de simplification, le discours cité n'étant pas encadré par des guillemets dans la plupart des énoncés, le DD mimétique est assimilé au DD canonique dans la suite de l'article. 
Anscombre $(2014,8)$, la "médiativité " correspond à "la mention de la source de l'information". Le verbe say met alors simplement en relation une donnée factuelle avec la source objective de cette donnée :

(25) Ten dead in Strasbourg train crash, reports say (TheGuardian.com $14 / 11 / 2015)$

Le verbe say voit son sémantisme réduit à celui d'un "verbe messager " et il pourrait être remplacé par un "introducteur médiatif ${ }^{6}$ comme according to :

(25a) According to some reports, ten people died in Strasbourg train crash

Par ailleurs, il serait envisageable de changer de type de discours rapporté, la proposition reports say pouvant alors constituer la proposition matrice d'un énoncé au discours indirect classique (DIC) :

(25b) Reports say ten people died in Strasbourg train crash

Dans cet énoncé au DIC, le verbe say conserve sa valeur médiative et l'"effet de factualisation" dû à la combinaison d'un locuteur métonymique et du verbe say rend possible l'emploi du verbe de parole classique comme verbe introducteur du contenu propositionnel de la complétive en THAT effacé. L'effacement de THAT dans cette reformulation au DIC de l'énoncé d'origine illustre la "porosité " existant entre ces deux formes syntaxiques de DR que sont le DD et le DIC, comme le montre De Mattia-Viviès (2006).

Quelle que soit la forme de discours rapporté choisie (DD ou DIC) pour présenter une donnée factuelle, un certain degré de "déconnexion" entre la forme et le sens demeure car le sujet inanimé n'est pas producteur de paroles au sens littéral du verbe say.

Le nombre pluriel associé au nom reports ne représente pas un paramètre significatif dans l'analyse menée. Si le nom report est employé, le raisonnement établi précédemment demeure valable mutatis mutandis :

(26) UK parents among 'the strictest in Europe', report says (Telegraph.co.uk 10/08/2015)

L'expression est empruntée à Charolles et Vigier $(2005,24)$. 
L'énoncé précédent reformulé au DIC pourrait être :

(26a) Report says UK parents are among 'the strictest in Europe'

De plus, pour les énoncés dans lesquels l'incise finale n'est que le support d'expression d'une valeur médiative ${ }^{7}$, l'ordre canonique sujet-verbe ou l'inversion semblent être utilisés de manière indifférenciée quand le verbe d'incise est say:

(27) Ditch the emotion and recognise economic migrants are a benefit, says report (Telegraph.co.uk 19/01/2016)

Toutefois, avec l'inversion, c'est le nom report qui est mis en relief en tant que dernier terme de l'énoncé, la source énonciative du contenu propositionnel étant dévoilée au dernier moment dans le processus de lecture de l'énoncé de la gauche vers la droite.

Une étude que nous avons menée à partir du moteur de recherche interne du site Telegraph.co.uk a permis de recenser 30 incises médiatives survey says dans le corpus contenant les articles publiés sur le site Internet jusqu'en 2016. Le SN peut parfois être plus étoffé et inclure le nom de l'organisme ayant réalisé ou commandité l'enquête :

(28) Credit conditions ease for business, BoE survey says (Telegraph.co.uk 02/04/2010)

(29) Interest rates will double by end of 2015, Treasury survey says (Telegraph.co.uk 25/12/2014)

(30) Young people would miss mobiles and web more than TV, Ofcom survey says (Telegraph.co.uk 20/04/2011)

Le nom survey dans sa collocation avec le verbe say peut se rencontrer avec l'inversion, avec 51 occurrences dénombrées sur le site Telegraph.co.uk, comme dans l'énoncé suivant :

(31) British accent is more attractive than French, says survey (Telegraph.co.uk 08/02/2015)

Une incise réduite à sa fonction pragmatique de support d'expression d'une valeur médiative sera appelée incise médiative. 
La présence d'un acronyme comme source énonciative endossant la responsabilité du contenu propositionnel tenant lieu de discours cité semble imposer l'inversion avec le verbe say, la source informationnelle étant identifiée avec une grande précision référentielle, davantage qu'avec un nom générique comme report ou survey:

(32) City is unaccountable, says TUC (TheGuardian.com 29/06/2009)

(33) BT risks losing ' $£ 1.25 \mathrm{bn}$ ' to cheaper competitors, says UBS (Telegraph.co.uk 12/02/2016)

Dans ces deux énoncés, les locuteurs métonymiques désignent respectivement la confédération syndicale britannique TUC et la banque suisse UBS.

\section{Autres verbes introducteurs de la valeur médiative}

De nombreux verbes hormis say peuvent apparaître dans les incises médiatives, comme le verbe suggest, par exemple, qui est l'intertitre de l'article précédemment cité en (26) :

(34) British parents are among the strictest in Europe with even the Germans more lenient than UK mothers and fathers, report suggests (Telegraph.co.uk 10/08/2015)

La recherche de la collocation suggests report sur le site Telegraph.co.uk ne fournit aucune occurrence. Il n'existe pas à proprement parler de contraintes syntaxiques qui interdisent l'inversion (suggests report) mais cette inversion n'est pas employée dans ce journal. Ce résultat est confirmé par une recherche menée dans le British National Corpus où la collocation suggests report n'est pas attestée dans la presse.

Ainsi, en présence d'un verbe comme suggest qui est généralement classé dans la catégorie des verbes " directifs " (Searle 1976), la subjectivité du journaliste paraît subsumée par les contraintes normatives d'usage.

En présence d'un autre verbe exercitif comme warn, l'ordre canonique et l'inversion sont pourtant employés de manière indifférenciée :

(35) Britain should protect itself against 'tyranny of Eurozone majority', warns report (Telegraph.co.uk 28/01/2016)

(36) More plastic than fish in the oceans by 2050, report warns (Telegraph.co.uk 19/01/2016) 
L'hésitation perceptible sur la forme de discours rapporté la plus apte à relayer une donnée factuelle est aussi présente avec l'emploi d'un verbe comme find:

(37) Children's knowledge of nature is dwindling, study finds (Telegraph.co.uk 28/04/2015)

L'incise study finds associe une production textuelle humaine à un verbe qui permet de mettre en lumière l'obtention d'une donnée présentée comme fiable car établie à partir d'une démarche scientifique. Le sens de l'énoncé est conservé avec l'introducteur médiatif according to (37a) ou quand l'énoncé emprunte la forme du DIC (37b) :

(37a) According to a study, children's knowledge of nature is dwindling

(37b) A study finds children's knowledge of nature is dwindling

Il semble possible d'envisager une désinscription graduelle de l'origine de la donnée factuelle en fonction du choix de la forme de discours rapporté employée pour présenter le contenu propositionnel. Celle-ci peut se matérialiser par une gradation dans le détachement syntaxique entre le contenu propositionnel et la source médiative. Alors que la subordination syntaxique dans (37b) matérialise le lien étroit entre la donnée factuelle et sa source objective, le lien devient moins marqué avec une "modalisation en discours second $\|^{8}$ dans (37a) et encore plus lâche dans (37). L'oscillation constante entre DD et DIC est rendue possible par la valeur médiative de l'incise déterminée par le locuteur métonymique : c'est une caractéristique prégnante de ces titres d'articles de presse.

La disparition complète du segment contextualisant n'est pas à exclure, si le journaliste met en avant seulement la donnée factuelle :

(37c) Children's knowledge of nature is dwindling

Avec l'emploi d'un verbe non factif comme claim, qui partage ce statut avec le verbe say selon Harris $(1974,629)$, le degré de liberté associé à l'ordre sujet-verbe dans l'incise est conservé :

8 L'expression modalisation en discours second est empruntée à Authier-Revuz (1992, 39). 
(38) Four in every five sets of traffic lights should be removed, report claims (Telegraph.co.uk 25/01/2016)

(39) Millions of middle class Britons drinking too much, claims report (Telegraph.co.uk 29/08/2011)

La mention du locuteur métonymique peut parfois être incomplète, par souci de concision du titre :

(40) 'Child' migrant who killed asylum centre worker is an adult, Swedish migration rules (Telegraph.co.uk 12/02/2016)

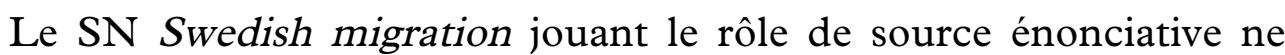
contient pas le nom recteur, qui est ici effacé. La lecture de l'article dévoile celui-ci : $S$ weden's migration agency.

\section{Énoncés localisés dans la zone de confluence de deux sous-ensembles}

L'étude minutieuse des énoncés contenant des incises médiatives en révèle le schéma compositionnel de base qui associe un syntagme nominal $X$ à un verbe introducteur $Y$.

\section{Les sous-ensembles du SN $X$ et du verbe introducteur $Y$}

Le SN $X$ est sélectionné dans une liste de sources énonciatives métonymiques à nombre singulier ou pluriel favorisant l'effet de factualisation :

$X=$ \{agency, analysis, council, figures, government, inquiry, newspaper,
organisations, papers, poll, report, research, source, stats, study,
survey, thinktank, watchdog... $\}$

Ces sources métonymiques ne sont pas homogènes dans leur composition sémantique: certaines désignent, par exemple, des noms collectifs (comme government et thinktank), d'autres des termes abstraits référant à l'objet produit (comme analysis et report).

À cette liste, il faut ajouter tous les acronymes désignant des associations, organisations, syndicats, etc. et les locuteurs métonymiques désignant des noms de pays, de villes, etc.

Dans une incise médiative, qui mime la composition syntaxique d'une occurrence de DD, le verbe doit tout d'abord faire partie des verbes 
LES TITRES D'ARTICLES DE PRESSE AU CONFLUENT DE PLUSIEURS

FORMES DE DISCOURS RAPPORTÉ

annonçant un discours cité. Une seconde condition, plus restrictive, est que ce verbe doit aussi être en mesure d'introduire du DIC, ce qui illustre la porosité déjà évoquée pour ces énoncés :

$Y=\{$ admit, announce, claim, find, predict, reveal, say, show, state, suggest, warn...\}

Voici quelques énoncés illustrant la réunion de ces deux conditions :

(41) 13 million abortions carried out every year in China, newspaper reveals (TheGuardian.com 30/07/2009)

(42) UK's most deserted train station had 14 passengers last year, figures reveal (Telegraph.co.uk 02/05/2013)

(43) Taiwan 'invaded' China, declassified papers show (Telegraph.co.uk 22/04/2009)

(44) Huge rise in sex offences on trains, official figures show (Telegraph.co.uk 20/08/2014)

(45) Labour will 'support HS2 if costs come down', sources claim (Telegraph.co.uk 29/10/2013)

(46) Upgrading existing rail network would be better value than HS2, government analysis finds (Telegraph.co.uk 03/11/2013)

(47) Ed Miliband's 'weak leadership' is swing voters' biggest concern, research shows (Telegraph.co.uk 12/08/2014)

(48) Rip up the busiest railways and replace them with bus lanes, says Thatcherite thinktank (Telegraph.co.uk 03/02/2015)

(49) Paris terror attacks not expected to put people off the city, travel organisations state (WesternDailyPress.co.uk 16/11/2015)

(50) Bobbies on the beat could vanish, warns police watchdog (Telegraph.co.uk 20/10/2015)

\section{Reconfiguration fonctionnelle au DIC}

Il a été établi précédemment que la structure de base d'un énoncé contenant une incise médiative est :

$P,(X, Y)$ ou $P,(Y, X)$ 
L'énoncé de départ peut être reconfiguré ainsi, l'incise médiative étant déplacée en position initiale dans l'énoncé pour devenir la proposition matrice :

$$
(X, Y)(T H A T) / T O P
$$

Alors que la proposition matrice médiative $(X, Y)$ est en tête de phrase, le contenu propositionnel $P$ occupe la position syntaxique de l'objet du verbe $Y$.

Lors de cette modification structurelle, le contenu propositionnel $P$, s'il est une proposition, peut faire l'objet d'une nominalisation complétive (45a) ou infinitive (48a):

(45a) Sources claim Labour will 'support HS2 if costs come down'

(48a) Thatcherite thinktank says to rip up the busiest railways and replace them with bus lanes

Ces locuteurs métonymiques n'étant pas des animés humains, un certain degré de "déconnexion forme/sens 》 voit le jour dans ces énoncés au DIC, comme l'évoque De Mattia-Viviès (2006).

De plus, les verbes de DD n'introduisant pas du DIC semblent exclus des incises médiatives comme les verbes d' "action-expression" (laugh, smile...) identifiés par Gournay (2015).

Par ailleurs, le degré de déconnexion forme/sens peut être élevé quand la source énonciative n'est pas directement en relation avec un animé humain :

(51) Life on Earth appeared hundreds of millions of years earlier than previously thought, ancient crystals show (Independent.co.uk 20/10/2015)

Les cristaux de roche ne portent pas en eux la notion sémantique de source énonciative. Pourtant, leur présence dans une proposition matrice médiative est rendue possible par une intervention humaine : des humains ont analysé ces cristaux et en ont tiré une hypothèse scientifique sur l'apparition de la vie sur la Terre. Un énoncé reformulé au DIC (51a) ainsi que l'emploi d'un introducteur médiatif (51b) incluant ce sujet inanimé sont tout à fait envisageables :

(51a) Ancient crystals show life on Earth appeared hundreds of millions of years earlier than previously thought 
(51b) According to ancient crystals, life on Earth appeared hundreds of millions of years earlier than previously thought

La recevabilité grammaticale de ces deux énoncés illustre une nouvelle fois la plasticité de la langue.

\section{Conclusion}

La présente étude a mis en évidence que l'ordre sujet-verbe dans une incise en position finale dans un titre d'article de presse est régi par différentes contraintes sémantiques et pragmatiques. Ainsi, la collocation entre un $\mathrm{SN} X$ et un verbe introducteur $Y$, qui sont les deux arguments constitutifs de l'incise, obéit à une multitude de règles d'usage.

En présence d'un verbe comme say ou d'autres verbes non factifs comme claim, la subjectivité du journaliste-locuteur rapporteur s'exprime à loisir, l'ordre canonique et l'inversion étant acceptables grammaticalement. C'est alors l'importance relative de la source informationnelle qui peut inciter le locuteur rapporteur à choisir l'ordre canonique ou l'inversion.

Dans le cas d'une incise médiative, au sein de laquelle un locuteur métonymique prend en charge un contenu propositionnel, une instabilité énonciative liée à l'identification de la forme de DR utilisée est mise au jour. Le titre d'article de presse apparaît ainsi au confluent de plusieurs formes de DR en concurrence (DD, DIC ou modalisation en discours second). Tout en épousant les contours énonciatifs d'une occurrence de DD mimétique, l'énoncé contenant une incise médiative illustre la porosité entre ces formes de DR car le verbe introducteur de DD qui la compose est aussi potentiellement un verbe introducteur de DIC. Cette instabilité énonciative repose sur la labilité et la plasticité de la langue, qui tend à résister à une catégorisation stricte et définitive.

Des recherches complémentaires sur cette thématique permettront probablement d'obtenir une modélisation encore plus fine des relations sémantiques et syntaxiques qui guident l'agencement sujet-verbe dans les titres d'articles de presse. 


\section{BIBLIOGRAPHIE}

\section{Articles De PRESSE}

The Daily Telegraph, sites Internet Independent.co.uk, Telegraph.co.uk, TheGuardian.com et WesternDailyPress.co.uk

\section{BASE DE DONNÉES}

British National Corpus

\section{OUVRAgES ET ARTICLES DE RÉFÉRENCE}

ANSCOMBRE, Jean-Claude. 2014. "Présentation ". In J.-C. ANSCOMBRE, E. OPPERMANN-MARSAUX \& A. RODRÍGUEZ SOMOLINOS (dir.), Médiativité, polyphonie et modalité en français. Paris : Presses Sorbonne Nouvelle, p. 7-16.

AUSTIN, John L. 1962. How To Do Things with Words. The William James Lectures delivered at Harvard University in 1955. Ed. J. O. URMSON. Oxford : Clarendon Press.

AUTHIER-REVUZ, Jacqueline. 1992. "Repères dans le champ du discours rapporté ». L’information Grammaticale 55 : 38-42.

BONHOMME, Marc. 1998. Les figures clés du discours. Paris : Seuil.

CHARAUDEAU, Patrick. 2006. "Discours journalistique et positionnements énonciatifs. Frontières et dérives ". In A. RABATEL \& A. CHAUVIN-VILENO (dir.), Énonciation et responsabilité dans les médias. SEMEN 22. Besançon: Presses Universitaires Franc-Comtoises, p. 29-43. 
CHARAUDEAU, Patrick. 2005. Les médias et l'information. L'impossible transparence $d u$ discours. Louvain-la-Neuve: De Boeck et Larcier/INA.

CHAROLLES, Michel \& VIGIER, Denis. 2005. "Les adverbiaux en position préverbale : portée cadrative et organisation des discours ». Langue française $148: 9-30$.

DE MATTIA-VIVIÈS, Monique. 2006. "De la porosité des formes du discours rapporté aux cas de déconnexion forme/sens dans l'univers $\mathrm{du}$ récit ». In C. DELESSE (dir.), Discours rapporté(s): approche(s) linguistique(s) et/ou traductologique(s). Arras: Artois Presses Université, p. 29-52.

DE MATTIA-VIVIÈS, Monique. 2010. «Du discours rapporté mimétique aux formes intrinsèquement hybrides ". SIGMA/ANGLOPHONIA $28: 151-180$.

GOURNAY, Lucie. 2015. "Les problèmes de traduction posés par l'articulation Discours Direct / Récit». E-rea 12.2 http://erea.revues.org/4211, consulté le 10 juin 2016.

HARRIS, Richard. J. 1974. "Memory and Comprehension of Implications and Inferences of Complex Sentences ». Journal of Verbal Learning and Verbal Behaviour 13 : 626-637.

LACAZE, Grégoire. 2011. "De l'incise au segment contextualisant : un changement d'horizon dans l'introduction du discours direct". Études de Stylistique Anglaise $1: 25-44$.

LACAZE, Grégoire. 2013. "Word order in utterances of direct speech in English: a subtle balance between conventions and innovation ". Erea $11.1 \mathrm{http}: / /$ erea.revues.org/3406, consulté le 10 juin 2016.

LACAZE, Grégoire. 2015. "L'énonciation aphorisante dans l'article de presse: une syntaxe sous contrôle(s)». E-rea 12.2 http://erea.revues.org/4200, consulté le 10 juin 2016. 
LACAZE, Grégoire. 2016. "Responsabilité et prise en charge énonciatives dans les titres d'articles de presse». Études de Stylistique Anglaise 9 : 31-57.

MAINGUENEAU, Dominique. 2012. Les phrases sans texte. Paris : Armand Colin.

SEARLE, John R. 1976. "A classification of illocutionary acts ». Language in Society $5.1: 1-23$. 